(e-migrinter

e-Migrinter

$14 \mid 2016$

L'ajustement méthodologique comme fabrique critique du savoir dans les études migratoires

\title{
La migration des jeunes dakarois comme horizon... La reconstruction d'un objet de recherche
}

\section{Kelly Poulet}

\section{OpenEdition}

\section{Journals}

Édition électronique

URL : https://journals.openedition.org/e-migrinter/732

DOI : 10.4000/e-migrinter.732

ISSN : 1961-9685

Éditeur

UMR 7301 - Migrinter

Référence électronique

Kelly Poulet, «La migration des jeunes dakarois comme horizon... La reconstruction d'un objet de recherche ", e-Migrinter [En ligne], 14 | 2016, mis en ligne le, consulté le 20 mai 2021. URL : http:// journals.openedition.org/e-migrinter/732 ; DOI : https://doi.org/10.4000/e-migrinter.732

Ce document a été généré automatiquement le 20 mai 2021.

Tous droits réservés 


\title{
La migration des jeunes dakarois comme horizon... La reconstruction d'un objet de recherche
}

\author{
Kelly Poulet
}

1 Durant six années, j'ai enquêté auprès d'une cinquantaine de jeunes dakarois et dakaroises qui énonçaient, tous et toutes, l'intention de «partir pour mieux revenir ». Ces jeunes sont nés et ont grandi au sein de trois quartiers de la capitale sénégalaise, Sacré-Cœur III, Hann-Marigot et Niaari-Tally, qui reflètent le visage pluriel de la ville et regroupe respectivement une population économiquement aisée, pauvre et intermédiaire. Cette hétérogénéité du visage de la capitale prend pour origine l'histoire même de sa construction et rend compte des vécus différenciés des jeunes selon les quartiers où ils se sont socialisés. Les situations économiques des familles se répercutent sur le mode de vie de ces jeunes qui ont dès lors des rapports différents à l'argent, à l'emploi et aux études, des activités et des temporalités distinctes, la jeunesse s'expérimentant selon des rythmes quotidiens divers d'un quartier à un autre. En cela, ces quartiers peuvent constituer une échelle et un prisme d'analyse pertinents pour étudier les aspirations à la migration des jeunes. Celle-ci, âgée au début de mon enquête de 19 à 30 ans, a été appréhendée moins sous l'angle d'une classe d'âge définie, que comme une catégorie sociale aux contours flous. Le fait d'être jeune est avant tout défini tel un statut, et au Sénégal, celui d'être considéré comme un "cadet social» (Awenengo d'Alberto, 2011), n'ayant pas encore franchi les étapes permettant d'accéder au statut d'adulte reconnu ${ }^{1}$ (Antoine, Razafindrakoto, Roubaud, 2001).

2 Ma recherche doctorale part ainsi du constat que l'ensemble des jeunes enquêtés aspirant à partir, considèrent la migration comme la meilleure perspective pour accéder à ce statut d'adulte reconnu. Elle cherche à rendre compte des aspirations de cette jeunesse urbaine hétérogène qui ne part pas - ou n'est pas encore partie -, appréhendées sous l'angle des processus de domination et d'émancipation qui se donnent à voir au Sénégal. En effet, des rapports sociaux de génération, de classe, de « caste » et de sexe contraignent le devenir désiré par ces jeunes qui n'occupent pas les 
mêmes positions dans ces rapports. La migration leur semble être le meilleur horizon des émancipations possibles. Pourtant, aucun d'entre eux n'est (encore) parti. Les trajectoires biographiques de ces jeunes et les rapports sociaux dans lesquels ils sont imbriqués donnent à voir quelles sont les (im)possibilités de sortir du territoire pour s'en sortir, nonobstant les contraintes qui les retiennent. La migration est restée en puissance ${ }^{2}$, tout au long de mes écrits et des cheminements personnels de ces jeunes.

3 Réussir à considérer le sens de cette migration comme horizon, à partir des aspirations à devenir de ces jeunes de Dakar, n'a pu l'être qu'au regard des interrogations suscitées par une pratique de terrain fragmentée dans le temps et dans l'espace, c'est-à-dire faites de retours réguliers à Dakar et de va-et-vient entre Paris, Amiens et Dakar. Cette approche m'a permis de me confronter aux regards et aux critiques des enquêtés, mettant en cause mes certitudes et mes pratiques sur le terrain.

4 Je reviendrai donc dans un premier temps sur le sujet que j'avais formulé aux prémices de mes recherches, ainsi que sur le dispositif méthodologique que j'avais élaboré. Dans un second temps, je présenterai la pratique de terrain qui a inscrit mon enquête dans ce que je dénomme le "nomadisme sédentaire ", et qui m'a permis d'assister à la reconstruction perpétuelle du devenir des jeunes enquêtés. Cette position n'a cessé de me contraindre à revisiter mon propre "projet de départ ", mon objet originel de recherche, et m'a amené à reconsidérer théoriquement que le projet de départ migratoire de ces jeunes enquêtés restait en suspens.

\section{De l'objet recherché}

5 C'est avec un objectif scientifique précis et un dispositif méthodologique adapté au sujet d'enquête que j'ai débuté mes recherches doctorales. Après deux années de recherche effectuées en master sur les causes des départs vers l'Europe de jeunes dakarois (Poulet, 2009, 2010), j'ai commencé ma recherche doctorale avec l'ambition d'appréhender, dans la capitale sénégalaise, les migrations de ces jeunes. J'envisageai de saisir le profil des migrants et leurs logiques migratoires ${ }^{3}$ (de départ et de retour) depuis le «vouloir partir » émergeant dans le pays de départ jusqu’à la concrétisation de leur projet puis dans le pays dit « d'accueil », et jusqu'à un éventuel retour au pays.

6 Les recherches récentes sur les phénomènes migratoires actuels s'accordent sur le fait que les migrations ont changé (Audebert, Ma Mung, 2007): elles ne sont plus synonymes d'un départ définitif depuis le pays d'origine, mais s'accompagnent de multiples retours, plus ou moins réguliers; les dynamiques, les flux et leurs compositions ont subi de profonds changements comme au Sénégal. L'expression de « nouvelle vague émigratoire sénégalaise » vient d'ailleurs désigner le tournant qu'ont pris les dynamiques migratoires de ce pays depuis les années 1990 (Tall, 2002; Fall, 2007). En effet, si la migration internationale sénégalaise a longtemps été l'apanage de populations rurales avec une zone de recrutement privilégiée, à savoir - la zone du fleuve Sénégal -, la région de Dakar fournit aujourd'hui l'essentiel des migrants du pays, notamment parce qu'elle abrite une part considérable de la population sénégalaise (Bouilly, Marx, 2008). Sur les 177273 émigrés recensés, 27,5\% proviennent de la région de Dakar (ANSD, 2013) et $12 \%$ des départements de la vallée du Fleuve Sénégal, Matam et Podor (Lessaut, Mezger, 2010). La population migrante se caractérise aussi par sa jeunesse $-68 \%$ des émigrés ont un âge compris entre 14 et 34 ans et $94,1 \%$ sont des émigrés actifs d'âge compris entre 15 et 54 ans (OIM, 2009). Elle s'est par ailleurs 
relativement féminisée, notamment au départ de Dakar. L'autre fait nouveau est l'émergence de pays de destination dépourvus de liens antérieurs, qu'ils soient historiques, culturels ou linguistiques, avec le Sénégal: l'Italie et l'Espagne, en l'occurrence, sont peu à peu devenues des destinations « montantes ", comme les ÉtatsUnis (Willem, 2007) ${ }^{4}$.

7 Nouveaux profils de migrants, flux inédits, pays de transit comme au Maghreb (Alioua, 2010), pays anciennement de destination devenus autant d'étapes vers un ailleurs : les phénomènes migratoires actuels sont appréhendés comme un processus caractérisé avant tout par la notion de mouvement, insistant sur la fluidité de la mobilité. Pour Chadia Arab (2009), la migration serait avant tout une histoire de mouvement et de déplacement, et une renégociation permanente des relations et des projets. C'est en ce sens qu'elle mobilise la notion de " circulation migratoire ». Ce concept présente selon la géographe l'avantage de mieux rendre compte, dans le contexte actuel de mondialisation migratoire, des processus de territorialisation et de valorisation spatiale différenciée qui s'en trouvent $a$ fortiori complexifiés. Certains chercheurs appréhendent alors le mouvement dans le déplacement et font de la mobilité un cadre d'enquête, voire une méthode (Peraldi, 2002; Tarrius, 2002; Brachet, 2012). Par exemple, Alain Tarrius (2002) adopte une approche de terrain nomade auprès de ce qu'il nomme des " transmigrants ", et évolue «du Maroc aux Balkans en passant par l'Espagne, le Sud de la France et la Turquie, et en cherchant à capter les moments et les lieux où ces mobilités transnationales et les territoires qu'elles dessinent s'entrecroisent, induisent des rencontres, et présagent des changements en cours » (Alioua, 2008, p. 18).

8 Pour ma part, c'est une vision plus «traditionnelle » du "faire du terrain » qui m'animait au début de ma thèse; une vision pourtant bousculée par ce renouveau théorique et méthodologique décrit ci-avant. Par "traditionnelle», j'entends une pratique élaborée au 20ème siècle, qui considère le terrain de recherche de manière "balisée ", tel un espace délimité et comme un moment suspendu où le chercheur part de manière prolongée pour enquêter. Cette «fixation » se pensait dans un temps long d'enquête. Dans cette conception, l'espace-temps de la fixation était une donnée indissociable d'un «bon » terrain qui se définissait donc dans l'unité de lieu. La qualité de l'ethnographie dépendant dans une certaine mesure de l'exhaustivité des données recueillies, elle est mesurée par l'ancrage. Ce dernier est pensé majoritairement dans la durée d'un séjour continu, et donc - en tant que spécificité des « hommes de terrain »dans la sédentarité. Les études migratoires en sociologie et en anthropologie n'ont pas échappé à cette conception du terrain, délimité spatialement et figé temporellement : les recherches se sont longtemps focalisées sur les espaces de départ ou sur les communautés d'immigrants dans les pays d'accueil, plus rarement sur les doubles espaces. Descriptions de lieux et/ou de moments fixes, ces travaux tentent de saisir, par ce biais, les phénomènes liés à la migration. Bien sûr, l'internationalisation des terrains a été une donnée non négligeable dans l'histoire de l'ethnologie ; pensons par exemple à Catherine Quiminal (1991), étudiant les foyers parisiens auprès des travailleurs maliens et remontant jusqu'aux communautés des bords du fleuve Sénégal. Toutefois, il n'en reste pas moins que la plupart des adeptes de cette dialectique multipolaire des terrains, dans les études migratoires, le faisait sur un temps très long, par succession de recherches au cours de leur carrière.

9 Afin de comprendre les processus migratoires des Dakarois et Dakaroises, j'ai ainsi suivi, dès les prémices de ma recherche, les prescriptions d'Abdelmalek Sayad (1999) : 
selon l'auteur, il fallait saisir le phénomène migratoire dans sa globalité à partir d'une dialectique spatiale. Et je retrouvais alors dans cette approche, la prise en compte des espaces multiples ou des "terrains fixes multisitués", selon l'expression de George Marcus (1995). Pour ma part, j'ai décidé d'articuler échelles spatiales et échelles sociales, comme je l'explique ci-après.

Du point de vue empirique, le "terrain» de recherche correspondait aux lieux de provenance et d'arrivée de jeunes travailleurs Dakarois âgés de 20 à 29 ans $^{5}$, récemment immigrés en France, auprès desquels j'ai réalisé mon mémoire de master 2. En France, mon aire géographique d'étude comprenait Paris, ses villes en banlieue et quelques villes proches géographiquement, telles que Beauvais et Amiens, ayant un accès ferroviaire direct à la capitale où le travail se "trouvait plus facilement ", d'après les enquêtés. Au Sénégal, les quartiers dakarois de provenance de ces immigrés ont délimité mon aire de terrain. J'ai tâché de m'insérer au plus près des familles des migrants enquêtés, en vivant au rythme du voisinage et des jeunes avec qui les migrants avaient nourri des rêveries collectives avant leur départ. Ces trois quartiers de la capitale sénégalaise sont ainsi devenus mes points de chute.

11 Je considérais méthodologiquement ces derniers comme autant d'«espace(s) historique(s) et social(aux) plus ou moins homogène(s) et singulier(s), [de] lieu(x) d'interactions relevant d'un vécu commun et faisant l'objet de représentations symboliques» (Ndione, Lalou, 2007, p. 240), et pouvant participer à la diffusion de comportements migratoires supposés particuliers. L'objectif était de saisir les influences à l'œuvre dans la volonté de migrer - ce que je nomme les " potentialités de départ » -, qui opèrent bien avant la migration effective ou même la formulation de son projet. Par la suite, il s'agissait aussi de saisir l'ensemble du processus de concrétisation du projet migratoire, en constante évolution, jusqu'au départ effectif.

12 Adoptant une démarche qualitative inspirée de l'ethnographie multisituée, il me fallait être dans un premier temps "ici ", en l'occurrence en France, auprès des migrants enquêtés, puis partir pour un temps donné «là-bas ", à Dakar, dans les quartiers de départ: rencontrer leur «nous social» de référence me permettait de comparer et d'objectiver les discours formulés par les migrants et d'observer, dans le quartier comme au sein de l'habitat familial, les conséquences directes, matérielles et symboliques de leur migration.

13 J'ai alors pensé mon dispositif méthodologique et ma posture sur le terrain, en termes d'ancrage spatial dans deux espaces, comme la meilleure perspective d'analyse des transformations et des circulations des migrants. Mais les temporalités imposées par les rythmes de l'institution universitaire, les obligations et les contraintes bureaucratiques, m'obligèrent finalement à revoir mon calendrier de terrain. Deux temps longs étaient difficiles à envisager et il me fallut les réduire. Une telle "fragmentation temporelle" me conférait alors une posture méthodologique faite d'allers-retours, de circulations entre Dakar et la France. Si cette configuration m'apparaissait au départ comme le propre d'une anthropologie à durée déterminée, m'empêchant de maîtriser véritablement la durée et les temporalités propres au rythme du terrain (cérémonies, étapes...), n'étais-je pas finalement encore plus proche de la configuration propre aux populations enquêtées, et in fine de mon objet d'étude? 


\section{...au « nomadisme sédentaire » comme outil d'enquête}

14 J'ai ainsi pensé contourner cette configuration contraignante en faisant de la circulation un outil méthodologique. N'étais-je pas en effet plus proche de la population et de ses rythmes propres, à suivre leurs mouvements, leurs allers-retours plus ou moins réguliers? N'allais-je pas prendre pleinement part, aux côtés des jeunes, aux nouvelles dynamiques de la mobilité, fluide et continue ? L'enthousiasme fut bref.

15 Je développais la posture d'une "nomade sédentaire » : nomade d'une part, par mes allers- retours continus entre Paris et Dakar, et sédentaire d'autre part, durant les moments où je m'immergeais à Dakar et en France au sein des quartiers des enquêtés. Je jouais de la mobilité et de la fixation, du déplacement et de l'immersion (Abélès, 2012). Durant les périodes de l'enquête, ma confrontation aux réalités du terrain - que je vais présenter dans un premier temps -, a par deux fois mis à plat les œillères théoriques, voire militantes, avec lesquelles j'avais pu appréhender les travaux dans lesquels je m'engageais. De part et d'autre des frontières, les jeunes dakarois m'ont fait prendre conscience de ma posture de «dominante», par mon inscription dans les rapports sociaux spatialisés, un point que j'analyserai dans un second temps.

\section{Ma confrontation en tant que « chercheure mobile » aux formes de domination}

16 En effectuant ces allers-retours entre Paris et Dakar, je suis devenue un liant du «nous social » de référence des migrants, ce « nous » qui englobe, dans ce continuum temporel, ceux « d'ici » et ceux de « là-bas». Cette position m'octroyait de nombreux avantages sur le terrain : je pouvais comparer les discours avec les conditions d'existence réelles. Je confrontais mes observations à la subjectivité des récits de vie récoltés de part et d'autre. Au delà de mes enquêtes réalisées des deux côtés de la frontière, je suis également devenue un enjeu. De simple transporteuse de bagages divers, dont le contenu par ailleurs me renseignait sur la nature des échanges et des cadeaux demandés et/ou envoyés depuis la France - lettres et photos -, d'autres requêtes m'ont peu à peu été adressées : on me demandait d'exercer un contrôle et de dresser un compte-rendu de ce qu'il pouvait bien "se passer » de l'autre côté de la frontière. Plus encore, mes va-et-vient étaient source de suspicion : qu'est-ce que tu vas dire de moi, de nous, d'ici, là-bas? Il y a certaines choses qu'il ne faut pas dévoiler, on a notre fierté, c'est peutêtre la seule chose qu'il nous reste encore! (Extrait d'entretien, 2010). Cette "fierté » des immigrés, à ne pas ébranler, et ces interrogations me renvoyaient à ma position de témoin qui circule dans le temps et dans l'espace. Ma présence auprès de ces migrants faisait en effet de moi un témoin de certaines formes de domination auxquelles ils pouvaient se retrouver confrontés, telles que la précarité des conditions de vie au sein des foyers de travailleurs ou des appartements que nombre d'entre eux partageaient dans la promiscuité. J'assistais surtout à leurs désillusions, leurs moments de colère, face à ce qu'ils appellent "les mensonges des anciens", soit de ceux partis avant eux (Poulet, 2014). À Dakar, j'assistais aux étapes, aux démarchages et aux stratégies pour pouvoir partir : je me confrontais aux jeunes dakarois et à l'échec de leurs démarches de sortie du territoire sénégalais, aux humiliations ressenties de la part des institutions, mais également aux pressions multiples exercées sur ces cadets sociaux, considérés comme des charges sociales par les familles, le voisinage (Timéra, 2001). 

ceux qui étaient restés, et d'autre part obligeait ces derniers à l'égard de ceux qui étaient partis. Chacun fait «bonne figure » de chaque côté de la frontière : d'un côté, les jeunes restés à Dakar face aux émigrés, dont on veut être l'égal, et de l'autre, les émigrés face à ceux dont on s'est distancé, mais desquels on attend de la considération. Mes premiers résultats montrent que le départ représente pour les jeunes autant une quête de prestige social et d'émancipation de sa condition de "jeune", que de ressources financières. La migration est alors pensée comme un moyen de reconnaissance sociale.

Pour autant, ce sont davantage mes prises de position face à ces non-dits qui ont été mises en cause par ceux-là mêmes qui m'avaient accueilli dans leur univers matériel et moral, et qui m'ont à cette occasion fait partager et vivre leurs expériences du mépris, du désir de s'émanciper, des stratégies de contournement des formes de domination et de contrôle, et des obligations.

\section{Une mise en cause de ma posture critique par les enquêtés}

Si j'ai très tôt considéré l'engagement ${ }^{6}$ auprès des enquêtés ainsi que ma part de subjectivité, ma position de liant entre les immigrés et leurs familles, et de personne qui circule, me confrontait à la complexité de l'univers moral des immigrés dans lequel j'étais intégrée et auquel je participais. Face aux désillusions de ceux arrivés, et aux espoirs de ceux restés, je pensais que "dire la vérité » sur les conditions de vie des immigrés en France pouvait contribuer à mettre en cause cet imaginaire migratoire qui pousse nombre de jeunes à considérer le départ vers le(s) «Nord(s) » comme l'unique horizon d'émancipation.

J'ai alors adopté une position ouvertement critique auprès des enquêtés, dont l'extrait de carnet de terrain ci-après rend compte.

\section{Extrait de carnet de terrain. 2012, Saint-Denis (France)}

Je me suis rendue à la colocation des Dakarois, dans un quartier de la banlieue parisienne. Aujourd'hui, plus que d'habitude, les conversations ont porté sur les stratégies mises en place pour ne pas « devoir dire non, mais ne pas à avoir à donner de l'argent » à ceux restés au pays. Boubacar vient de changer de numéro de téléphone, pour la troisième fois. Abdoulaye, quant à lui, a prétexté ce mois-ci qu'il avait déjà envoyé de l'argent pour d'autres raisons - à qui ? À d'autres que sa famille directe ? Face à ces tentatives de contournements des envois d'argent d'une part, et face aux critiques envers celles et ceux, qui au Sénégal, « ne comprennent pas que c'est dur ici » d'autre part, je me suis exclamée :

Pourquoi ne pas leur dire la vérité? Que tu ne peux pas donner! Il ne faudrait pas plutôt expliquer et montrer comment sont traités les Sénégalais ici en France? Montre-leur ce que tu fais exactement de tes journées, dis-leur que tu bosses tout le temps pour un salaire dérisoire! Peut-être que si tous les Sénégalais faisaient ça, ils [les autres au pays] ne voudraient pas partir, non?

Je ne cachais ainsi pas mon point de vue sur les subtilités de langage et les non-dits qui pouvaient entourer leur expérience migratoire. Et mes prises de position, 
apparaissant sûrement naïves à certains, furent parfois mises en accusation ou contrecarrées par les enquêtés en France :

$\mathrm{Tu}$ as beau dire les choses, ils ne comprennent pas, ils ne veulent rien entendre. Laisse-moi gérer comme je le peux. C'est facile pour toi. Tu n'as rien à prouver, tu circules comme tu veux, tu n'as pas les pressions administratives ni les obligations familiales et de réussite! Quand tu vas là-bas, tu as déjà réussi pour les gens, tu viens de France et tu es une tubaab! (Une blanche).

21 À Dakar, j'adoptais similairement une posture critique face à cette représentation sociale répandue chez les jeunes, et selon laquelle « là-bas, c'est mieux qu'ici », comme le met en évidence le carnet de terrain ci-après :

\section{Extrait de carnet de terrain. 2012, Niaari-Tally (Dakar).}

Je suis allée à Niaari-Tally aujourd'hui. Je n'ai pas pu m'empêcher de critiquer ouvertement ce que les jeunes s'imaginent de la France. Toutes celles et tous ceux présents aujourd'hui chez Kiné dénonçaient le comportement de sa sœur, Aby, présente en France depuis quelques années : elle « n'a rien construit ici »; elle « délaisse ses sœurs »; « l'argent qu'elle envoie n'est pas suffisant pour entamer des projets ici »; « elle ne fait pas d'efforts pour qu'elles aussi puissent partir ». Face à cette discussion sur des airs d'accusation, j'ai alors donné des détails sur les conditions de vie telles que je me les figure en France : j'ai mis en avant les difficultés auxquelles Aby pouvait être confrontée en France, comme celle de trouver un emploi. Je déclarais quel était le salaire moyen en France, et je décrivais quelles étaient les charges que nous pouvions avoir chaque mois à payer. Je tentais de persuader mes interlocuteurs de la véracité des expériences douloureuses et difficiles que ma trajectoire de militante avait pu rencontrer, au-delà de mon seul parcours universitaire.

J'ai ainsi tenté, en jouant de ma position de " connaisseuse ", de convaincre ces jeunes que les représentations pouvaient être sujettes à bon nombre d'interprétations pouvant se révéler elles-mêmes illusoires et ainsi décevantes. Ma posture critique a, de nouveau, été mise en accusation :

Tu as beau nous dire ce que tu veux! Toi tu connais là-bas, tu as vécu là-bas, tu as grandi là-bas, tu as fait le tour. Aujourd'hui tu es venue chercher des choses ici à Dakar. Tu y travailles, tu as découvert beaucoup de choses, tu as grandi. Maintenant tu aimes ici et tu viens et retournes là-bas comme tu veux. Nous on veut aussi avoir cette chance-là, découvrir, voir par nous-mêmes! Tout ce que l'on nous interdit de voir, alors que toi tu peux circuler comme tu veux. Nous on veut circuler, comme toi tu le fais!

Loin de tout onirisme social, c'est dans une conversation ordinaire, comme nous pouvions en avoir régulièrement, que Youssou m'a fait cette déclaration. Il n'a pas été le seul. D'autres enquêtés ont pu, d'une autre manière, m'exposer ce point de vue. L'exercice de poser par écrit tant de maladresse et de naïveté de ma part a été pourtant bien nécessaire. La portée de mes discours était bien évidemment limitée par ma position de "privilégiée ". J'incarnais, de leurs points de vue, ce monde en mouvement auquel les jeunes dakarois aspirent, et j'étais ainsi associée à ceux qui ont la place la 
mieux lotie, celle de ceux qui bougent et qui circulent sans pression, qu'elles soient d'ordre matériel, administratif ou moral.

Le point de départ de mon enquête, la France, et ma position de "nomade sédentaire ", que je pensais similaire à celle des migrants, m'avaient de prime abord fait considérer que la France était le point d'arrivée, l'aboutissement spatial du projet de mes interlocuteurs. Cependant, leurs propos vis-à-vis de ma posture critique me permirent finalement de réaliser que ce pays, tout comme l'Espagne ou l'Italie, n'était qu'une étape parmi d'autres dans leurs projets. L'aboutissement du projet est, pour les candidats à l'émigration, le point de départ : Dakar. Le retour est une condition de leur projet, puisque la réussite se fera dans la reconnaissance, là-bas, de ceux dont on s'est distancé, chose que l'on pourra apprécier, sur place. Le retour n'est pas forcément pensé comme définitif : partir pour revenir, partir et revenir... pour repartir et revenir.

Ce «nomadisme sédentaire» s'est finalement confirmé comme un outil méthodologique me conférant un statut qui, critiqué et envié par les enquêtés, rendait compte de la «réalité d'une planète divisée» (Augé, 2009, p. 13). Cette réalité, je l'observais à Paris et à Dakar avant ceux qui restent, ces «immobiles involontaires " (Carling, 2002). En effet, la situation administrative de nombre d'immigrés à Paris ne leur permettait ni de quitter le territoire ni de revenir à Dakar comme ils le souhaitaient. Et dans la capitale sénégalaise, ceux qui ont l'esprit tourné vers le(s) « Nord(s)» ne pouvaient y accéder aisément.

Ces derniers, candidats à l'émigration et qui ne partent pas, sont majoritaires. C'est une majorité que mes allers-retours permettaient d'identifier comme des "sédentaires forcés ». Cette découverte latérale, au cœur d'une logique de fonctionnement autre et ciblée sur la circulation, m'a permis de recentrer mes recherches au sein du lieu d'aboutissement de l'hypothétique migration, du possible départ : Dakar.

\section{" Je suis la seule "qui voyage", ici » : la « migration en puissance » comme nouvel objet}

Obnubilée par mon objet d'enquête initial qui portait sur "l'expérience » de la migration - que je nomme la «migration en actes»-, j'avais omis tout un pan de la recherche dans ce champ d'étude, de même que certaines pistes de recherche sur le terrain d'enquête: précisément, ceux qui restent et qui sont nourris par l'idée du départ. Je n'avais pas pris conscience que, pour les candidats à la migration à Dakar, cette dernière est restée une hypothèse - jusqu'à ce jour - pensée comme la meilleure perspective pour atteindre ses horizons d'émancipation. Les jeunes des trois quartiers de la capitale, et que j'ai enquêté, font partie de ces deux tiers de la population mondiale qui n'ont pas le droit de circuler librement dans un monde en perpétuel mouvement - un monde globalisé - et qui se voient ainsi contraints à la fixation (Agier, 2008). J'ai noté, dans mon dernier carnet de terrain, le moment précis où je me suis confrontée à la nécessité de reformuler alors mon objet de recherche :

Extrait de carnet de terrain. 2014, Dakar.

Il me faut arrêter les enquêtes. Il me faut écrire la thèse maintenant. J’ai prévenu les jeunes du quartier aujourd'hui, qui me demandaient si mes recherches avançaient. J'ai ri un peu. Mes recherches ont sans doute avancé, - oui, mais eux ? 
Personne n'est parti. Un des jeunes m'a demandé « au fait, sur quoi tu travailles déjà ? ». Je crois que c'est la dixième fois que je le leur dis, et chacun oubli. Ils me perçoivent comme une touriste particulière, comme une expatriée, pour beaucoup, une tubaab (blanche) qui habite ici, à la " sénégalaise ", mais temporairement, pour " ses vacances ». Bref. Je lui réponds : j'écris une thèse sur les migrations des jeunes de Dakar. Cette phrase est devenue le moyen de couper court à la discussion, en France comme au Sénégal. Pas aujourd'hui. Amadou m'a rétorqué : mais... tu fais aussi tes enquêtes auprès de nous? Pourtant personne ne migre jusqu'à présent, et dans les autres quartiers, ils y arrivent? Tu devrais plutôt travailler sur nos galères pour voyager! (rires). Cette remarque aussi spontanée soit-elle, remet bel et bien en question cette obsession pour l'étude de ceux qui partent et fait suite aux critiques énoncées durant tout le cheminement de l'enquête. Je suis la seule qui porte avec elle et en elle, la migration en actes : je suis la seule « qui voyage ", ici. de l'absence de mouvement spatial et de la contrainte à «l'immobilité involontaire ». Cette fixation peut faire l'objet de tentatives de contournement, en réaction à l'assignation au pays d'origine : mes multiples accompagnements chez des "passeurs " m'ont fait intégrer également ce marché des migrations qui s'organise (Bernardot, 2012). Ce monde en mouvement peut ainsi être subi : la présence, la circulation ou même l'absence des autres sont des éléments importants qui engendrent en négatif le sentiment d'être soi-même, en étant soumis à ce que j'ai nommé le " départ passif », en ce qu'il peut avoir de frustrant pour celui qui est condamné à rester. J'ai alors développé la perspective selon laquelle la migration pouvait aussi exister à travers son absence. Contraints à l'ancrage, les jeunes dakarois, sur lesquels j'ai recentré mon regard, sont à l'image du "Survenant » de Germaine Guèvremont (1945), qui voit son espace clos durant un moment de sa vie, mais son temps, incertain et aléatoire, restant, lui, ouvert sur l'avenir. Au terme de six années de suivi de ces jeunes, j'ai pu constater qu'aucun d'entre eux n'est encore parti du Sénégal. Le processus migratoire étant un phénomène évolutif, ces candidats à la migration se verront peut-être propulsés sur les routes, un jour ou l'autre. Dans tous les cas, l'hypothèse du départ anime encore aujourd'hui chacun des esprits des enquêtés. C'est en cela que j'ai pu distinguer la « migration en actes » - correspondant à l'expérience physique du départ en migration - de la «migration en puissance»; cette dernière renvoie à la possibilité de concrétisation du départ qui, parce qu'elle reste incertaine, continue de nourrir, de hanter le quotidien des jeunes, et charge de sens leurs perspectives d'avenir, et oriente in fine l'agir de ces jeunes. En ce sens, la « migration en puissance » n'est pas abstraite de l'expérience de la migration. Ainsi, il ne s'agit pas de rompre avec la compréhension du phénomène migratoire en tant que tel, mais plutôt de le penser selon la perspective de ceux qui restent et qui ne sont pas encore partis, et qui ne partiront peut-être jamais - ceci à partir de cette jeunesse dont les projections quant à l'à-venir sont contraintes (Antoine, Razafindrakoto, Roubaud, 2001). 


\section{Conclusion}

Ce qui est devenu l'objet de ma thèse - à savoir, appréhender les trajectoires des jeunes dakarois qui ne partent pas -, n'a pu l'être que par l'adoption de la démarche de recherche du «nomadisme sédentaire ", m'ayant permis de me confronter aux regards et aux critiques des enquêtés. Ce dispositif méthodologique m'a permis de pénétrer l'intimité des logiques et des pratiques de la « sélection » qui s'opère entre les jeunes. J'ai pu comprendre l'évolution des situations, des discours, des envies et des stratégies déployées face aux injonctions, comme autant d'éléments pour saisir les processus migratoires que j'observais. Si "l'hypothèse migratoire ", mise en récit, présente une dimension individuelle et subjective, elle ne peut pour autant se réduire à l'individu. Ce dernier s'insère dans un groupe plus ou moins large et dans un contexte historique et social, qu'il faut prendre en considération.

L'analyse des expériences des jeunes dakarois, et de leur intentionnalité face à leur statut de "candidats au départ contraints à la fixation", permet de dépasser et de réconcilier une approche sociologique dualiste des phénomènes migratoires. Celle qui met l'accent plutôt sur les acteurs et celle qui présupposerait le poids des déterminismes sociaux sur l'individu, se rencontrent ici pour travailler de concert. Ainsi, à partir des trajectoires et des expériences des jeunes dakarois enquêtés, il s'agit de saisir leurs aspirations selon leurs dispositions sociales pour comprendre en quoi la migration est pensée comme le meilleur horizon d'émancipation possible pour «s'en sortir». Comme l'affirme Sylvie Mazzella, "l'intentionnalité individuelle ne se construit pas en opposition aux collectifs (État, réseau, famille), ni par étapes successives (de l'individu à l'État en passant par des cercles sociaux intermédiaires) mais en coproduction " (2014, p. 78). Nécessairement, l'intentionnalité, au cœur des expériences de vie des jeunes dakarois, suppose de prendre en compte le poids des conditions et des contraintes qui pèsent sur ces candidats au départ et sur leur capacité à rationaliser les choix qu'ils font. Ces choix dépendent des dispositions sociales des individus, de leur capacité à accomplir leur autonomie, à négocier pour agir sur les normes sociales et à faire face aux différentes contraintes qu'ils subjectivisent. La "migration en puissance », matérialisée dans la (re)formulation perpétuelle d'une possible migration, nous informe sur ce qui est de l'ordre du " pouvoir migrer ».

\section{BIBLIOGRAPHIE}

Abélès, Marc (2012) Anthropologie de la globalisation, Paris, Payot \& Rivages, 2ème édition, 304 p. (Petite Bibliothèque Payot).

Agence Nationale de la Statistique et de la Démographie (2013) Chapitre II : La migration internationale, in Situation économique et sociale du Sénégal en 2011, Dakar, ANSD, pp. 42-60.

Agier, Michel (2008) Gérer les indésirables, Paris, Flammarion, 349 p.

e-Migrinter, 14 | 2016 
Alioua, Mehdi (2008) À la rencontre de la sociologie d'Alain Tarrius. Du paradigme de la mobilité au territoire circulatoire, e-Migrinter, $\mathrm{n}^{\circ} 1$, pp. 7-19.[Disponible sur Internet]

Alioua, Mehdi (2010) Jeunes transmigrants subsahariens au Maroc, in Lorcerie, F. (Ed.) Pratiquer les frontières. Jeunes migrants et descendants de migrants dans l'espace franco-maghrébin, Paris, CNRS Éditions, pp. 59-82.

Antoine, Philippe ; Razafindrakoto, Mireille ; Roubaud, François (2001) Contraints de rester jeunes ? Évolution de l'insertion dans trois capitales africaines : Dakar, Yaoundé, Antananarivo, Autrepart, vol. 18, $\mathrm{n}^{\circ}$ 2, pp. 17-36.

Arab, Chadia (2009) Les Aït Ayad. La circulation migratoire des Marocains entre la France, l'Espagne et l'Italie, Rennes, PUR, $351 \mathrm{p}$.

Audebert, Cédric; Ma Mung, Emmanuel (Eds.) (2007) Les nouveaux territoires migratoires : entre logiques globales et dynamiques locales, Bilbao, Humanitarian Net; Université de Deusto, 308 p.

Augé, Marc (2009) Pour une anthropologie de la mobilité, Paris, Payot \& Rivages, 96 p.

Awenengo d'Alberto, Séverine (2011) Sénégal : les nouvelles formes de mobilisations de la jeunesse, Carnets du CAP, n 15, pp. 37-65.

Bernardot, Marc (2012) Captures, Vulaines-sur-Seine, Éditions du Croquant, 192 p. (Kriticos). Bouilly, Emmanuelle ; Marx, Nina (Eds.) (2008) Migration et Sénégal. Pratiques, discours et politique, Asylon(s), n³, [réf. du 22 janvier 2016] [Disponible sur Internet].

Brachet, Julien (2012) Géographie du mouvement, géographie en mouvement. La mobilité comme dimension du terrain dans l'étude des migrations, Annales de géographie, vol. 687-688, $n^{\circ} 5$, pp. 543-560.

Carling, Jorgen (2002) Migration in the age of involuntary immobility, Journal of Ethnic and Migration Studies, vol. 28, $n^{\circ}$ 1, pp. 5-42.

Fall, Papa Demba (2007) La dynamique migratoire ouest-africaine entre ruptures et continuités, Communication à l'atelier "Understanding Migration Dynamics in the Continent", Accra, Ghana, University of Ghana, 18-21st september, 24 p. [réf. du 22 janvier 2016] [Disponible sur Internet]. Guèvremont, Germaine (1945) Le Survenant, Montréal, Beauchemin, 215 p.

Lessault, David ; Mezger Cora (2010) La migration international sénégalaise. Des discours publics à la visibilité statistique, MAFE Working paper, $\mathrm{n}^{\circ}$ 5, $15 \mathrm{p}$. [Disponible sur internet].

Marcus, George (1995) Ethnography in/of the World System : The Emergence of multi-sited Ethnography, Annual review of anthropology, $\mathrm{n}^{\circ}$ 24, pp. 95-117.

Mazzella, Sylvie (2014) Sociologie des migrations, Paris, Presse Universitaire de France, 128 p.

Ndione, Babacar ; Lalou, Richard (2007) Tendances récentes des migrations internationales dans le Sénégal urbain : existe-t-il une dynamique de quartier ? Les exemples de Dakar, Touba et Kaolack, in Association Internationale des Démographes de Langue Française (Ed.) Les migrations internationales : observation, analyse et perspectives. Colloque international de Budapest (20-24 septembre 2004), Paris, Presses Universitaires de France ; AIDELF, pp. 239-258.

Organisation Internationale pour les Migrations (2009) Migration au Sénégal. Profil national 2009, Dakar, OIM, $142 \mathrm{p}$.

Peraldi, Michel (Ed.) (2002) La fin des norias? Réseaux migrants dans les économies marchandes en Méditerranée, Paris, Maisonneuve \& Larose, 495 p. 
Poulet, Kelly (2009) La migration sénégalaise : dynamique migratoire entre rupture et continuité. Essai sur les causes des départs par pirogue des jeunes de Hann-Yarakh, village urbain de Dakar, vers les îles Canaries, Amiens, Université de Picardie Jules Verne, $119 \mathrm{p}$.

Mém.Master 1 : Sociologie : Amiens : 2009.

Poulet, Kelly (2010) Les migrations sénégalaises contemporaines : quelle place pour l'individu? Une approche biographique du processus d'individualisation au travers de la mobilité, Amiens, Université de Picardie Jules Verne, $155 \mathrm{p}$.

Mém.Master 2 : Sociologie : Amiens : 2010.

Poulet, Kelly (2014) De Paris à Dakar, discours croisés et enjeux autour de la mémoire de l'expérience migratoire, Asylon(s), nº 12 [réf. du 22 janvier 2016] [Disponible sur Internet].

Quiminal, Catherine (1991) Gens d'ici, gens d'ailleurs. Migrations soninké et transformations villageoises, Paris, Christian Bourgeois, $222 \mathrm{p}$.

Sayad, Abdelmalek (1999) La double absence. Des illusions de l'émigré aux souffrances de l'immigré, Paris, Seuil, 438 p.

Tall, Sérigne Mansour (2002) L'émigration internationale Sénégalaise d'hier à demain, in Diop, M.-C. (Ed.) (2002) La société sénégalaise entre le local et le global, Paris, Karthala, pp. 549-578.

Tarrius, Alain (2002) La mondialisation par le bas. Les nouveaux nomades de l'économie souterraine, Paris, Balland, $170 \mathrm{p}$.

Timéra, Mahamet (2001) Les migrations des jeunes sahéliens : affirmation de soi et émancipation, Autrepart, vol. 18, n², pp. 37-49.

Willem, Roos (2007) Barcelona or the Here after : Senegalese struggling with perilous journeys and perilous livelihoods, Kolor: Journal on Moving Communities, vol. 7, n 1, pp. 23-47.

\section{NOTES}

1. Les jeunes de Dakar se voient actuellement «contraints de rester jeune ", c'est-à-dire qu'ils n'accèdent que difficilement à un emploi, au mariage ; des étapes clés pour accéder au statut d'adulte. C'est ici une perspective retenue en sociologie de la jeunesse, qui associe l'entrée dans la vie adulte au franchissement d'étapes majeures marquant une rupture avec les états antérieurs à celles-ci. Dans ce contexte, la famille, principal filet de sécurité sociale, prend en charge ses membres inactifs en âge de travailler et peine à les considérer autrement que telle une charge financière.

2. Dans l'usage qu'en fait Aristote, ce qui n'est qu'en puissance, par opposition à ce qui est en acte, est ce qui n'est pas encore réalisé, ce qui n'est qu'une virtualité. Elle représente l'indéterminé et le possible.

3. Par logiques migratoires, j'entendais alors étudier les déterminants, les causes et les stratégies de départs et de retours.

4. Il est difficile aujourd'hui de mesurer l'actualité de ce schéma. La crise de ces dernières années a frappé de plein fouet ces deux pays.

5. J'excluais alors les immigrés pour étude dans cette recherche car je m'intéressais principalement aux personnes caractérisant la «nouvelle vague migratoire sénégalaise», composée majoritairement de jeunes hommes partant pour travailler et non par le biais de visas pour étude. Aussi, si le mobile économique était mis en avant par ces jeunes, je voulais alors approfondir cette raison évoquée en considérant les ressorts plus existentiels, comme la volonté 
de s'affirmer individuellement, qui m'était apparue une année plus tôt auprès de jeunes qui avaient tenté d'émigrer en pirogue vers les îles Canaries, rapatriés à Dakar.

6. Par « engagement " j'entends ici à la fois ma position de militante auprès de sans-papiers qui m'a fait intégrer le milieu des immigrés sénégalais en France, mais également la position de confidente, de «transmetteuse " d'informations et de cadeaux, de «conseillère » et de régulatrice de conflits familiaux.

INDEX

Index géographique : Sénégal, Dakar

Mots-clés : jeunes, projet migratoire, libre circulation, enquête, méthodologie, Sénégalais

\section{AUTEUR}

\section{KELLY POULET}

Doctorante en Sociologie, CURAPP-ESS (UMR 7319)

kelly.poulet@gmail.com 